\title{
A Simulated Annealing approach for solving Minimum Manhattan Network Problem
}

\author{
S. M. Ferdous \\ Ahsanullah University of Science and Technology(AUST) \\ Dhaka
}

\author{
Anindya Das \\ Iowa State University, Ames \\ Iowa
}

\begin{abstract}
In this paper we address the Minimum Manhattan Network (MMN) problem. It is an important geometric problem with vast applications. As it is an NP-complete discrete combinatorial optimization problem we employ a simple metaheuristic namely Simulated Annealing. We have also developed benchmark datasets and tested our algorithm with the dataset.
\end{abstract}

\section{General Terms:}

Experimental Algorithms, Stochastic Approach

\section{Keywords:}

Combinatorial Optimization, Metaheuristics, Simulated Annealing, Network Length

\section{INTRODUCTION}

Finding minimum network length is an important problem in computer science. In this paper we address the problem of finding minimum network length in Manhattan metric. Given two points $p, q \in \mathbb{R}^{2}$, a rectilinear path is achieved between these two points if all the line segments along the path is either vertical or horizontal. To a find a Manhattan path between $p$ and $q$ two properties must be satisfied:

- The path between $p$ and $q$ must be rectilinear.

- The length of the path is exactly equals to $\operatorname{dist}(p, q)=\| p . x-$ $q . x\|+\| p . y-q . y \|$.

In MMN problem, we are given a set $T$ of $n$ points in $\mathbb{R}^{2}$. A network $M$ is said to be manhattan network on $T$, if for all $p, q \in T$, there exist at least one Manhattan path between $p$ and $q$ with all its edge segment on $M$. Minimum Manhattan Network problem is to find the Manhattan Network $M$ with minimum network length.

MMN has vast applications in geometric network design as well as in VLSI circuit design, where the (rectilinear) characteristics of Manhattan networks adapt well to reality. An important example is computer chip manufacturing where all the circuit paths are usually rectilinear paths. Furthermore, the MMN problem has its applications in city planning, network layout and distributed algorithms [9].
MMN has its application in the field of computational biology. In [14], the author present a solution to the problem of designing efficient search spaces for pair hidden Markov models that align biological sequences using Manhattan networks.

\section{LITERATURE REVIEW}

The MMN problem has various applications already discussed in the previous section. Therefore, researchers started to work with this problem. Gudmundsson et al. [8] first published an $O\left(n^{3}\right)$ time 4 -approximation algorithm. Moreover, they proposed an $O(n \lg n)$ time 8-approximation algorithm and also discussed the problem of determining the complexity class of this problem.

Gudmundsson et al. [8] conjectured that there could be a 2approximation algorithm. Kato et al. [11] proved them right by providing an $O\left(n^{3}\right)$ time 2-approximation algorithm, although using a different approach. The key idea provided by them is to determine efficiently whether a graph is a Manhattan Network or not. A naive approach is to check whether Manhattan path exists for all $O\left(n^{2}\right)$ pairs of nodes, but they proved that it is sufficient to check only $O(n)$ specific pair of points. Using similar idea as [11], Benkert et al. [1] proposed a 3-approximation algorithm which runs in $O(n \lg n)$ time and takes linear space.

Benkert et al. [2] also proposed a mixed integer programming formulation. Chepoi et al. [4] used the idea of Pareto front and stripstaircase decomposition to derive a rounding 2-approximation algorithm based on an LP-formulation of the problem. Later, Guo et al. [9] proposed a 2-approximation algorithm based on dynamic programming which runs in $O\left(n^{2}\right)$ time. They also proposed another approximation algorithm with same approximation ratio, but with a better running time of $O(n \lg n)$ using a simple greedy strategy [10]. Seibert et al. [15] provided a 1.5-approximation algorithm.

So far the complexity class of this problem remained unknown. Chin et al. [5] first proved that Minimum Manhattan Network in 2 dimension is strongly NP-complete by reducing $3-S A T$ to this problem. It is not known whether this problem is APX-hard or not. For 3 dimension, Munoz et al. [13] proved this problem to be NPhard. They proposed a 3-approximation algorithm which is the first approximation algorithm in 3 dimension for a restricted version of this problem. 
First approximation algorithm for Generalized Minimum Manhattan Network was proposed by Das et al. [6]. For an arbitrary dimension $d$, they proposed an algorithm with approximation ratio $O\left(\lg ^{d+1} n\right)$.

\section{BASICS OF SIMULATED ANNEALING (SA)}

Simulated Annealing (SA) is a generic probabilistic algorithm and it is sometimes commonly said to be the oldest among the metaheuristics. It is also one of the first algorithms that had an explicit strategy to escape from local minima. The name and inspiration come from annealing in metallurgy, a technique that involve heating and controlled cooling of a material. Heating and cooling the material affects both the temperature and the thermodynamic free energy. While the same amount of cooling brings the same amount of decrease in temperature it will bring a bigger or smaller decrease in the thermodynamic free energy depending on the rate that it occurs, with a slower rate producing a bigger decrease.

This notion of slow cooling is implemented in the Simulated Annealing algorithm as a slow decrease in the probability of accepting worse solutions as it explores the solution space. SA was first presented as a search algorithm for Combinatorial Optimization problems in [7] 12]. The basic idea is to permit moves that result in solutions of worse quality than the current solution (uphill moves) to escape from local minima. The probability of doing such a move is decreased during the search which is controlled by the temperature parameter. The high level algorithm is described in Algorithm (1).

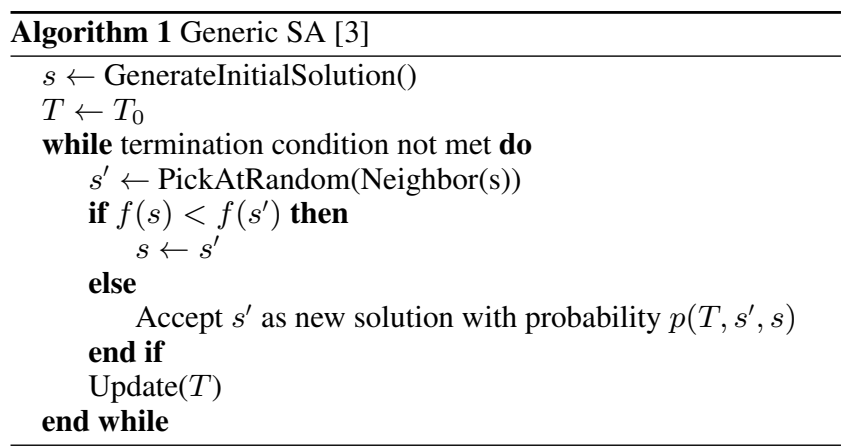

\section{OUR APPROACH: SIMULATED ANNEALING FOR MMN}

In this section, we will describe the SA implementation for MMN in details. The high-level pseudocode for solving MMN by SA is shown in Algorithm (2).

The algorithm starts with initializing a set of parameters. After reading the dataset it starts with generating an initial solution $(s)$. Then at each iteration it selects a new solution $\left(s^{\prime}\right)$ by tweaking the previous one and it is accepted as new current solution depending on size $(s)$, size $\left(s^{\prime}\right)$ and $T$ where size $(S)$ is the fitness(the manhattan network size) of solution $S$ and $T$ is the temperature parameter. As it is described in [3], we will use the Boltzman distribution computed as $\exp \frac{-\left(\operatorname{size}\left(s^{\prime}\right)-s i z e(s)\right)}{T}$ to find the probability of selecting a worse solution $\left(p\left(T, s^{\prime}, s\right)\right)$. The temperate, $T$ is decreased at each iteration using the equation $T_{i}=\alpha \times T_{i-1}$, where $\alpha \in[0,1]$.

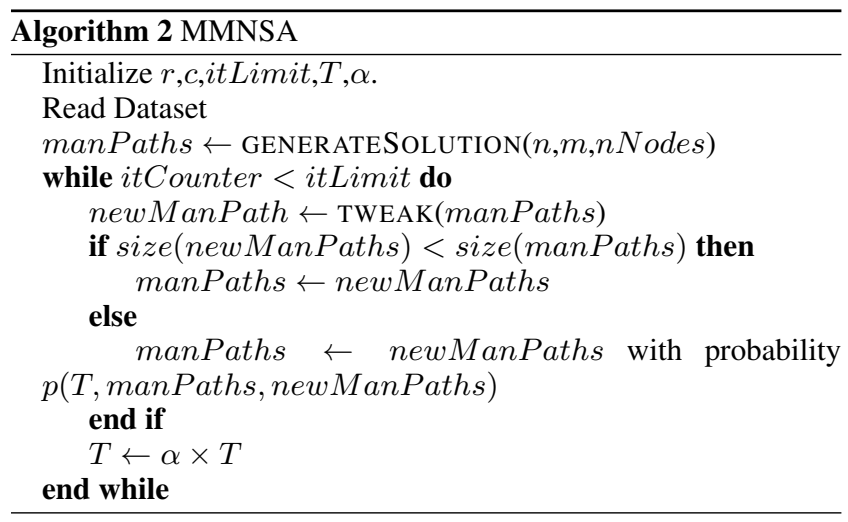

\subsection{Generating a solution}

For each pair of nodes in the grid, we construct a probabilistic manhattan path. The manhattan network is constructed from all the individual manhattan paths.

We have developed a stochastic approach to construct a manhattan path between two nodes which will be called source and destination nodes henceforth. The algorithm is iterative in nature. Starting from the source node at each step the algorithm chooses a feasible grid edge randomly from a uniform distribution. Then the source node is updated to the other end of the chosen edge. The procedure is continued until the source becomes destination. From a source node it is necessary to detect the feasible grid edges. The feasible set of edges from a particular source node depend on the orientation of the source and destination nodes. The possible orientation and the feasible edges are shown in Figure (1). The detailed pseudocode of generating a solution is shown in Algorithm (3)

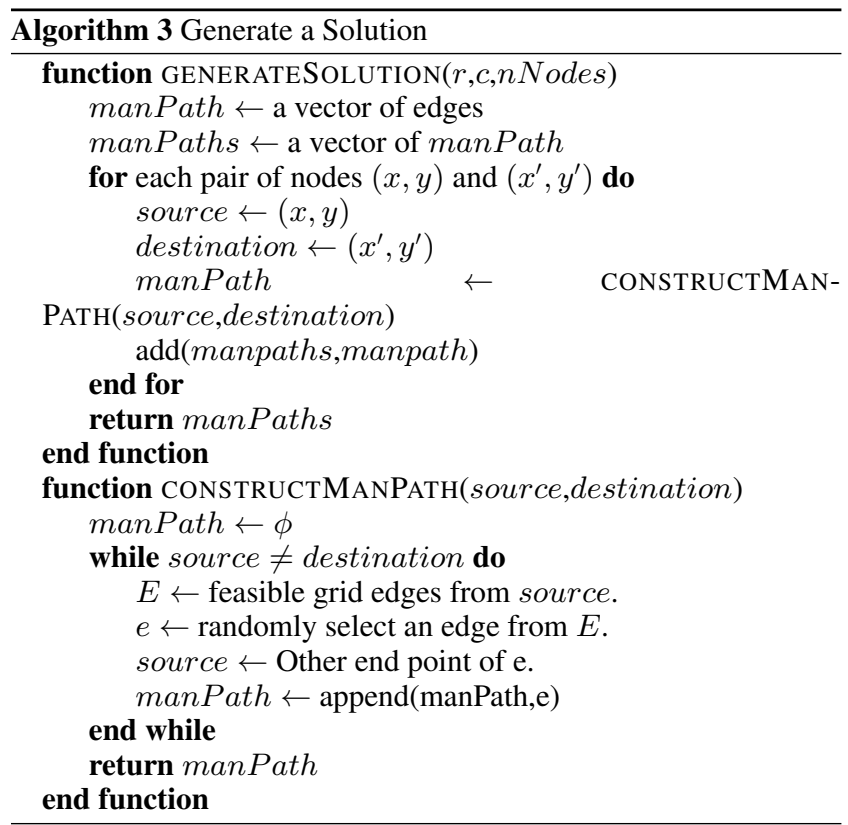




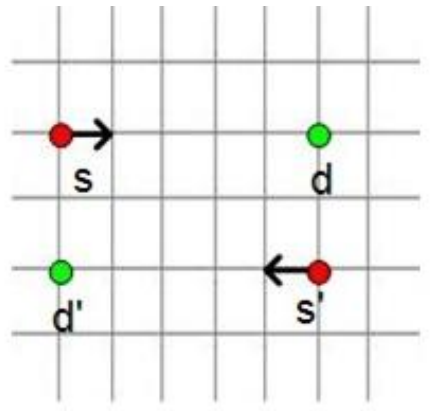

(a)

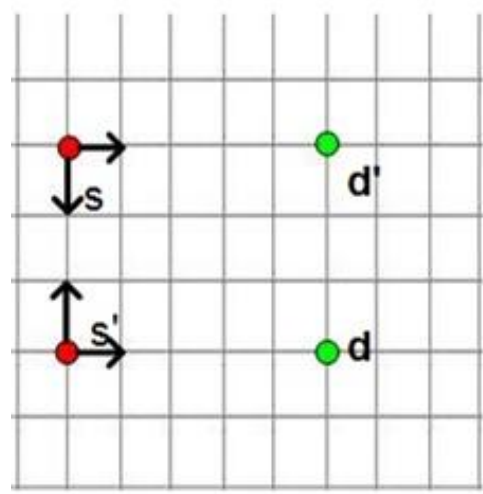

(c)

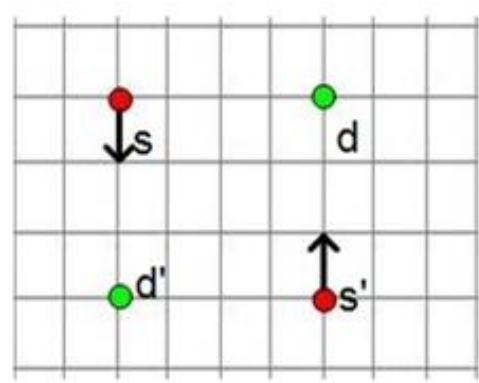

(b)

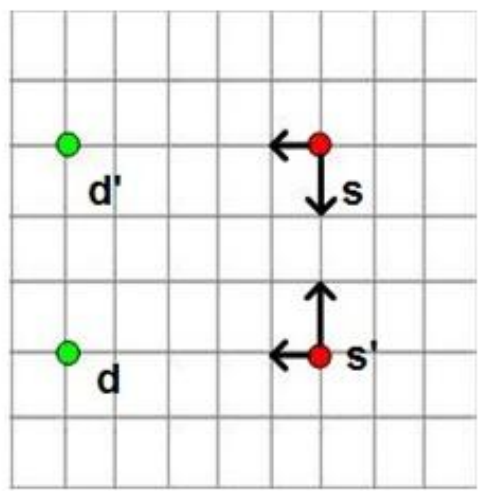

(d)

Fig. 1. Feasible grid edges for different source and destination orientation $\left((\mathrm{s}, \mathrm{d})\right.$ and $\left.\left(\mathrm{s}^{\prime}, \mathrm{d}^{\prime}\right)\right)$. The bold arrows are the allowed edges from the source node. (a)-(b): Orientations where one edge is feasible. (c)-(d): Orientations where two edges are feasible.

\subsection{Tweaking a solution}

Tweaking is the process of generating new solutions given any valid solution. To get a new solution we randomly select a pair of nodes. Then we reconstruct the manhattan path between these two nodes. The detailed pseudocode is given in Algorithm (4).

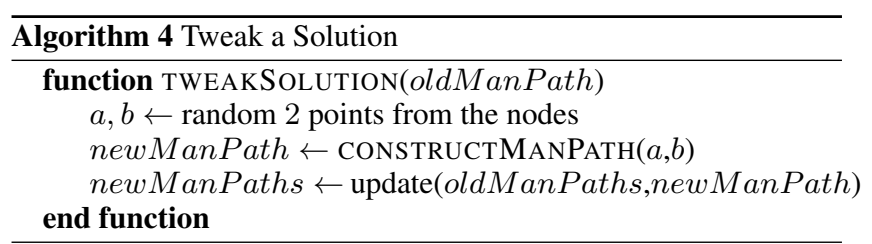

\section{EXPERIMENTS}

We have conducted our experiments in a computer with Intel Core 2 Quad CPU $2.33 \mathrm{GHz}$. The available RAM was $4.00 \mathrm{~GB}$. The operating system was Windows 7 . The programming environment was Matlab.

\subsection{Datasets}

To our best knowledge, there are not any benchmark dataset for MMN. Here we introduced a random set of data. We set the grid size $(r \times c)$ as $20 \times 20$, i.e. both the number of rows and columns are 20 . We divide the datasets into four groups. Each group contain 10 test cases. For group 1 each test case contain 10 points. 25, 50 and 100 points are considered in the each test cases of group 2 , group 3 and group 4 respectively.

\subsection{Results}

Table 13 represent the results of SA algorithm. The three tables are generated for 3 cooling values. The result is divided in 4 groups. Under each group we have 2 columns. The first column represent the average of 10 independent runs of the algorithm. The second column is the standard deviations of the runs. 


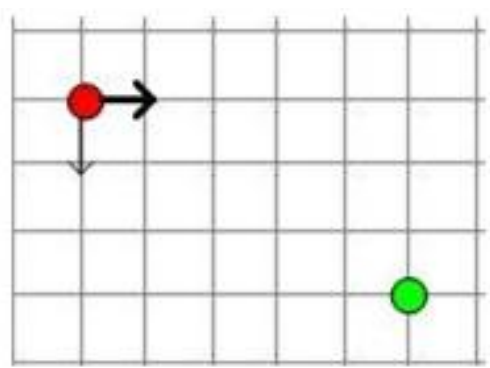

(a)

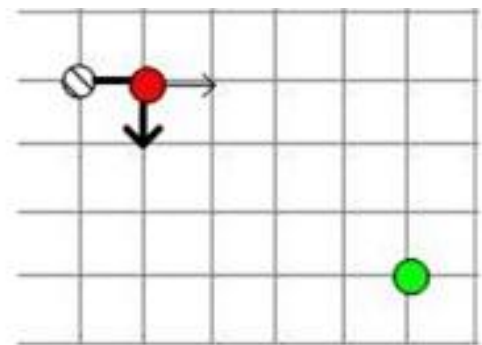

(b)

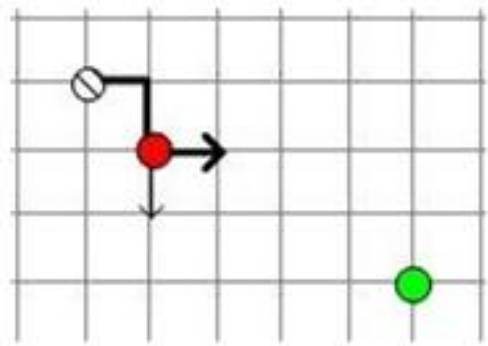

(c)

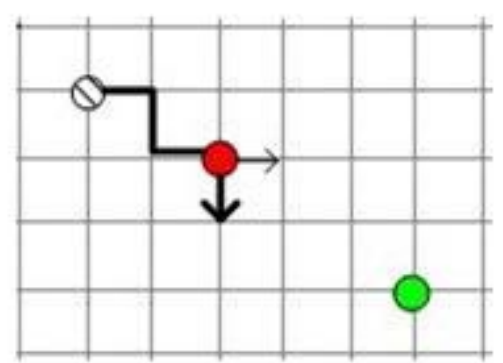

(d)

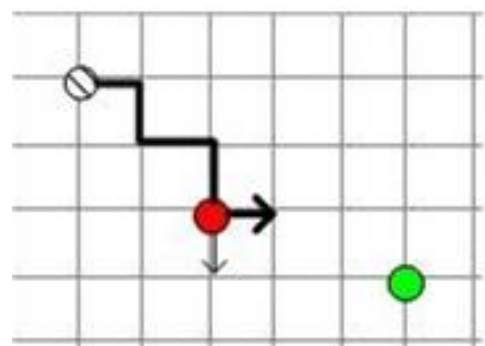

(e)

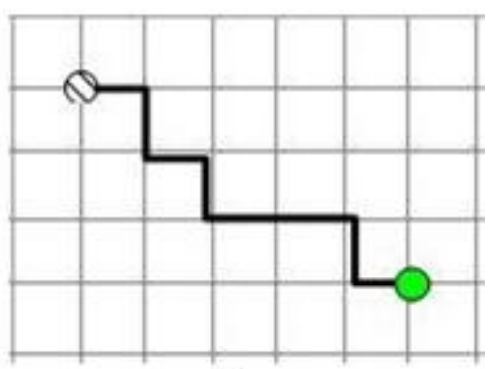

(f)

Fig. 2. Generating a solution. (a): feasible grid edges (arrowed) from the source node. Bold arrow is the selected edge. (b): update source according the selected grid edge. (c) - (e): continue selection of feasible grid edges and updating source. (f): Final Manhattan Path

\section{CONCLUSION}

In this paper we have developed a metaheuristic technique namely Simulated Annealing for solving the MMN problem. We have also developed several benchmark data sets. With these we have reported our findings and results. Future research might be in de- signing more metaheuristic approaches for the problem. A detailed comparative analysis of performances of different metaheuristics on this problem would be a good future exercise. 
Table 1. Network length by MMNSA $(\alpha=0.2)$

\begin{tabular}{|l|l|l|l|l|l|l|l|l|}
\hline group1 & \multicolumn{2}{l|}{ group2 } & \multicolumn{2}{l|}{ group3 } & \multicolumn{2}{l|}{ group4 } \\
\hline Avg. Net. Length & Std & Avg. Net. Length & Std & Avg. Net. Length & Std & Avg. Net. Length & Std \\
\hline 201.80 & 9.04 & 543.60 & 18.22 & 638.30 & 8.04 & 758.20 & 1.32 \\
205.60 & 15.58 & 556.50 & 7.82 & 661.10 & 6.76 & 756.50 & 2.80 \\
210.50 & 9.41 & 563.40 & 11.77 & 650.20 & 9.84 & 753.10 & 2.18 \\
251.10 & 9.87 & 552.10 & 8.27 & 705.50 & 6.55 & 754.80 & 2.86 \\
304.20 & 7.64 & 516.20 & 14.86 & 739.40 & 4.30 & 752.00 & 3.23 \\
205.10 & 10.95 & 523.50 & 17.60 & 724.90 & 6.98 & 748.60 & 2.99 \\
216.80 & 11.73 & 515.20 & 13.93 & 699.70 & 4.50 & 739.40 & 5.02 \\
174.20 & 11.55 & 479.30 & 7.62 & 712.40 & 7.09 & 753.20 & 2.53 \\
210.70 & 6.60 & 442.10 & 10.20 & 718.10 & 5.43 & 756.20 & & 1.93 \\
228.00 & 11.43 & 542.80 & 7.98 & 679.90 & 6.90 & 753.50 & & 2.42 \\
\hline
\end{tabular}

Table 2. Network length by MMNSA $(\alpha=0.5)$

\begin{tabular}{|l|l|l|l|l|l|l|l|}
\hline \multicolumn{2}{|l|}{ group1 } & \multicolumn{2}{l|}{ group2 } & \multicolumn{2}{l|}{ group3 } & \multicolumn{2}{l|}{ group4 } \\
\hline Avg. Net. Length & Std & Avg. Net. Length & Std & Avg. Net. Length & Std & Avg. Net. Length & Std \\
\hline 200.30 & 8.35 & 538.60 & 10.30 & 635.10 & 7.88 & 757.80 & \\
206.60 & 8.37 & 552.30 & 10.57 & 657.50 & 7.71 & 755.90 & 1.23 \\
205.90 & 12.65 & 554.50 & 12.89 & 654.30 & 7.51 & 753.20 & 3.11 \\
256.40 & 13.79 & 554.50 & 14.52 & 707.40 & 6.13 & 756.30 & 2.30 \\
307.00 & 7.83 & 519.40 & 13.72 & 738.50 & 3.57 & 753.00 & 1.34 \\
194.50 & 9.74 & 515.80 & 14.95 & 721.30 & 7.56 & 750.10 & 2.83 \\
208.20 & 5.71 & 514.20 & 9.80 & 698.80 & 6.01 & 738.70 & 3.98 \\
164.80 & 11.09 & 477.50 & 7.95 & 713.40 & 8.26 & 752.40 & 4.27 \\
208.50 & 4.40 & 439.50 & 14.28 & 718.60 & 5.58 & 756.30 & 2.95 \\
224.80 & 13.89 & 537.80 & 10.14 & 679.50 & 5.91 & 752.60 & 1.16 \\
\hline
\end{tabular}

\section{REFERENCES}

[1] Marc Benkert, Alexander Wolff, and Florian Widmann. The minimum manhattan network problem: A fast factor-3 approximation. In Proceedings of the 2004 Japanese Conference on Discrete and Computational Geometry, JCDCG'04, pages 16-28, Berlin, Heidelberg, 2005. Springer-Verlag.

[2] Marc Benkert, Alexander Wolff, Florian Widmann, and Takeshi Shirabe. The minimum manhattan network problem: Approximations and exact solutions. Comput. Geom. Theory Appl., 35(3):188-208, October 2006.

[3] Christian Blum and Andrea Roli. Metaheuristics in combinatorial optimization: Overview and conceptual comparison. ACM Comput. Surv., 35(3):268-308, September 2003.

[4] Victor Chepoi, Karim Nouioua, and Yann Vaxès. A rounding algorithm for approximating minimum manhattan networks. Theor. Comput. Sci., 390(1):56-69, January 2008.

[5] Francis Y.L. Chin, Zeyu Guo, and He Sun. Minimum manhattan network is np-complete. In Proceedings of the Twentyfifth Annual Symposium on Computational Geometry, SCG '09, pages 393-402, New York, NY, USA, 2009. ACM.

[6] Aparna Das, Krzysztof Fleszar, Stephen G. Kobourov, Joachim Spoerhase, Sankar Veeramoni, and Alexander Wolff. Polylogarithmic approximation for generalized minimum manhattan networks. CoRR, 2012.

[7] V. ern. Thermodynamical approach to the traveling salesman problem: An efficient simulation algorithm. Journal of Optimization Theory and Applications, 45(1):41-51, 1985.

[8] Joachim Gudmundsson, Christos Levcopoulos, and Giri Narasimhan. Approximating a minimum manhattan network. Nordic J. of Computing, 8(2):219-232, June 2001.
[9] Zeyu Guo, He Sun, and Hong Zhu. A fast 2-approximation algorithm for the minimum manhattan network problem. In Rudolf Fleischer and Jinhui Xu, editors, Algorithmic Aspects in Information and Management, volume 5034 of Lecture Notes in Computer Science, pages 212-223. Springer Berlin Heidelberg, 2008.

[10] Zeyu Guo, He Sun, and Hong Zhu. Greedy construction of 2approximation minimum manhattan network. In Proceedings of the 19th International Symposium on Algorithms and Computation, ISAAC '08, pages 4-15, Berlin, Heidelberg, 2008. Springer-Verlag.

[11] Ryo Kato, Keiko Imai, and Takao Asano. An improved algorithm for the minimum manhattan network problem. In Proceedings of the 13th International Symposium on Algorithms and Computation, ISAAC '02, pages 344-356, London, UK, UK, 2002. Springer-Verlag.

[12] S. Kirkpatrick, C. D. Gelatt, and M. P. Vecchi. Optimization by simulated annealing. SCIENCE, 220(4598):671-680, 1983.

[13] Xavier Muoz, Sebastian Seibert, and Walter Unger. The minimal manhattan network problem in three dimensions. In Sandip Das and Ryuhei Uehara, editors, WALCOM: Algorithms and Computation, volume 5431 of Lecture Notes in Computer Science, pages 369-380. Springer Berlin Heidelberg, 2009.

[14] Lior Pachter and Fumei Lam. Picking alignments from (steiner) trees. In Proceedings of the Sixth Annual International Conference on Computational Biology, RECOMB '02, pages 246-253, New York, NY, USA, 2002. ACM.

[15] Sebastian Seibert and Walter Unger. A 1.5-approximation of the minimal manhattan network problem. In Proceedings of 
Table 3. Network length by MMNSA $(\alpha=0.8)$

\begin{tabular}{|l|l|l|l|l|l|l|l|}
\hline group1 & group2 & \multicolumn{2}{l|}{ group3 } & \multicolumn{2}{l|}{ group4 } \\
\hline Avg. Net. Length & Std & Avg. Net. Length & Std & Avg. Net. Length & Std & Avg. Net. Length & Std \\
\hline 202.50 & 5.21 & 539.50 & 7.72 & 638.70 & 9.18 & 758.40 & 1.26 \\
210.70 & 9.74 & 544.60 & 9.94 & 661.30 & 6.96 & 757.00 & 1.70 \\
207.70 & 17.47 & 567.10 & 7.29 & 650.90 & 8.18 & 755.20 & 2.39 \\
252.70 & 6.18 & 548.30 & 15.31 & 707.50 & 1.72 & 756.60 & 1.90 \\
304.90 & 9.95 & 512.30 & 13.97 & 735.60 & 3.92 & 754.10 & 2.60 \\
205.10 & 10.40 & 517.70 & 11.42 & 725.50 & 5.21 & 748.90 & 2.38 \\
213.00 & 9.09 & 512.30 & 15.04 & 701.30 & 4.52 & 740.50 & 3.66 \\
175.60 & 8.09 & 468.90 & 12.41 & 717.00 & 5.85 & 753.00 & 1.56 \\
214.30 & 11.55 & 437.20 & 13.21 & 714.10 & 6.40 & 755.70 & 2.21 \\
226.90 & 8.57 & 539.00 & 11.37 & 679.00 & 8.96 & 751.60 & 2.67 \\
\hline
\end{tabular}

the 16th International Conference on Algorithms and Computation, ISAAC'05, pages 246-255, Berlin, Heidelberg, 2005.

Springer-Verlag. 